\title{
ON R-DUALS OF TYPE III IN HILBERT SPACES
}

\author{
HARTMUT FÜHR, JAHANGIR CHESHMAVAR AND ALI AKBARNIA \\ Hartmut Führ, Lehrstuhl A für Mathematik, RWTH Aachen University, D-52056 \\ Aachen, Germany \\ fuehr@matha.rwth-aachen.de \\ Jahangir Cheshmavar, Department of Mathematics, Payame Noor University, P.O.Box \\ 19395-3697, Tehran, Iran \\ j_cheshmavar@pnu.ac.ir \\ Ali Akbarnia, Department of Mathematics, Payame Noor University, P.O.Box \\ 19395-3697, Tehran, Iran \\ Aliakbarnia7@gmail.com
}

\begin{abstract}
Following work by Casazza, Kutyniok and Lammers, and extensions by Stoeva and Christensen, we provide some novel characterizations of $R$-dual sequences of type III in Hilbert spaces. We systematically extend the construction procedure by basing it on a choice of an antiunitary involution. For certain classes of $R$-duals of type $I I I$, we derive a representation of the associated frame operator in terms of spectral measures.
\end{abstract}

Key words and phrases. Frames, Riesz sequence, Riesz basis, Spectral representation, R-dual of type $I$, R-dual of type $I I I$.

2010 Mathematics Subject Classification: Primary 42C15; Secondary 53C05. 


\section{INTRODUCTION}

In this paper we consider frame/Riesz sequence properties for a sequence $\left\{f_{i}\right\}_{i \in I}$ in a Hilbert space $\mathcal{H}$ and the corresponding sequence depending on two orthonormal bases, the so-called $R$-dual sequence (Riesz-dual sequence) $\left\{\omega_{j}\right\}_{j \in I}$ generated by combined action of some operators on one of this orthonormal bases. Sequences of this form were introduced in [1] by Casazza, Kutyniok and Lammers with the purpose of deriving duality principles for frames in arbitrary separable Hilbert space $\mathcal{H}$. For each sequence in $\mathcal{H}$, they construct a corresponding sequence depending on the choice of two orthonormal bases, with a kind of duality relation between them, and then use them to derive duality principles for frames. They called this sequence $R$-dual sequence. $R$-dual sequences have since been considered by several authors, see $[5,6,7]$. One of the most important extensions for the current paper appears in the work of Stoeva and Christensen, which introduces various classes of $R$-duals, for example with the motivation to obtain general versions of the duality principle in Gabor frames [8]. In [7], the authors give an equivalent condition of two sequences to be $R$-duals.

In this paper we present variations of these constructions, that are based on choices of certain isometric operators on $\ell^{2}(I)$ that help implement the duality principles. Each choice of operator gives rise to a construction of $R$-dual sequences that have the same desirable properties as the original construction in [1], relating frame/Riesz sequence properties of the sequence $\left\{f_{i}\right\}_{i \in I}$, to properties of its $R$-dual $\left\{\omega_{j}\right\}_{j \in I}$, as studied in [1], but also by Christensen and Kim in [5] and Stoeva and Christensen in [8]. Finally, given a type III of $R$-dual associated of a frame, we derive a representation for its frame operator, (see Theorem 4.4) via the spectral theorem. The main results appear in Section 3 and Section 4. Section 2 contains some basic definitions and results.

\section{Preliminaries}

In what follows, we will review basic definitions of frame and Riesz basis and present type $I$ and $I I I$ of $R$-duals; for more details, we refer the interested reader to the $[4,3,1]$. Throughout this paper, let $\mathcal{H}$ be a separable Hilbert spaces, $I_{\mathcal{H}}$ the identity operator on $\mathcal{H}$ and $I$ be a countable index set. Also let $F:=\left\{f_{i}\right\}_{i \in I}$ and $\Omega:=\left\{\omega_{j}\right\}_{j \in I}$.

A collection of vectors $F$ in $\mathcal{H}$ is a Bessel sequence if there exists a constant $B>0$ so that

$$
\sum_{i \in I}\left|\left\langle f, f_{i}\right\rangle\right|^{2} \leq B\|f\|^{2},
$$

for all $f \in \mathcal{H}$. If in addition, there is a constant $A>0$ so that

$$
A\|f\|^{2} \leq \sum_{i \in I}\left|\left\langle f, f_{i}\right\rangle\right|^{2},
$$

for all $f \in \mathcal{H}$, then $F$ is a frame for $\mathcal{H}$. The constants $A$ and $B$ are called frame bounds. The frame $F$ is $A$-tight, if $A=B$. If at least the upper bounds $B$ exists, $F$ is called a Bessel sequence. Here, the synthesis operator of $F$ is defined by

$$
T_{F}: \ell^{2}(I) \rightarrow \mathcal{H} ; T_{F}\left\{c_{i}\right\}_{i \in I}=\sum_{i \in I} c_{i} f_{i} .
$$

Given a frame $F$ in $\mathcal{H}$, its frame operator is

$$
S_{F}:=T_{F} T_{F}^{*}: \mathcal{H} \rightarrow \mathcal{H} ; \quad S_{F} f=\sum_{i \in I}\left\langle f, f_{i}\right\rangle f_{i},
$$


where $T_{F}^{*}$ is the adjoint of $T_{F}$, and it is given by $T_{F}^{*} f=\left\{\left\langle f, f_{i}\right\rangle\right\}_{i \in I}$. In this case $S_{F}$ is a bounded, invertible, self-adjoint and positive operator. Moreover the sequence $\widetilde{F}:=$ $\left\{S_{F}^{-1} f_{i}\right\}_{i \in I}$ is also a frame for $\mathcal{H}$ satisfying the reconstruction formula $f=\sum_{i \in I}\left\langle f, S_{F}^{-1} f_{i}\right\rangle f_{i}$, for every $f \in \mathcal{H}$. The sequence $\widetilde{F}$ is called the canonical dual frame of $F$. Also, any sequence $G:=\left\{g_{i}\right\}_{i \in I}$ in $\mathcal{H}$ which is not the canonical dual and satisfies $f=\sum_{i \in I}\left\langle f, f_{i}\right\rangle g_{i}=$ $\sum_{i \in I}\left\langle f, g_{i}\right\rangle f_{i}$ is called an alternate dual frame of $F$.

A collection of vectors $\Omega$ in $\mathcal{H}$ is a Riesz sequence if there exist constants $C, D>0$ such that

$$
C \sum_{j \in I}\left|c_{j}\right|^{2} \leq\left\|\sum_{j \in I} c_{j} \omega_{j}\right\|^{2} \leq D \sum_{j \in I}\left|c_{j}\right|^{2},
$$

for all finite sequences $\left\{c_{j}\right\}_{j \in I}$. The numbers $C$ and $D$ are called Riesz bounds. A Riesz sequence $\Omega$ is a Riesz basis for $\mathcal{H}$ if $\overline{\operatorname{span}} \Omega=\mathcal{H}$.

We are now ready to introduce the main definitions considered in this paper. We begin with the following well-known properties:

- $F$ is a Bessel sequence in $\mathcal{H}$ if and only if $T_{F}^{*}$ is a well-defined and bounded operator on $\mathcal{H}$.

- $F$ is a frame for $\mathcal{H}$ if and only if $T_{F} o T_{F}^{*}: \mathcal{H} \rightarrow \mathcal{H}$ is a bounded and invertible operator.

- $F$ is a Riesz sequence in $\mathcal{H}$ if and only if $T_{F}^{*} o T_{F}: \ell^{2}(I) \rightarrow \ell^{2}(I)$ is a bounded and invertible operator.

Definition 2.1. Let $\left\{e_{i}\right\}_{i \in I}$ and $\left\{h_{i}\right\}_{i \in I}$ be orthonormal bases for $\mathcal{H}$. Let $\left\{f_{i}\right\}_{i \in I} \subset \mathcal{H}$ be such that $\sum_{i \in I}\left|\left\langle f_{i}, e_{j}\right\rangle\right|^{2}<\infty, \forall j \in I$. In [1] the $R$-dual of $\left\{f_{i}\right\}_{i \in I}$ with respect to the orthonormal bases $\left\{e_{i}\right\}_{i \in I}$ and $\left\{h_{i}\right\}_{i \in I}$ is defined as the sequence given by

$$
\omega_{j}=\sum_{i \in I}\left\langle f_{i}, e_{j}\right\rangle h_{i}, \quad j \in I .
$$

This R-dual is called of type $I$ in [8]. In what follows, we will review type $I I I$ of R-duals, which are essential to our main result in the next section.

Definition 2.2. [8] Let $F$ be a frame for $\mathcal{H}$ with frame operator $S_{F}$. Let $\left\{e_{i}\right\}_{i \in I}$ and $\left\{h_{i}\right\}_{i \in I}$ denote orthonormal bases for $\mathcal{H}$ and $Q: \mathcal{H} \rightarrow \mathcal{H}$ be a bounded bijective operator with $\|Q\| \leq \sqrt{\left\|S_{F}\right\|}$ and $\left\|Q^{-1}\right\| \leq \sqrt{\left\|S_{F}^{-1}\right\|}$. The R-dual of type III of $F$ with respect to the triple $\left(\left\{e_{i}\right\}_{i \in I},\left\{h_{i}\right\}_{i \in I}, Q\right)$, is the sequence $\Omega$ defined by

$$
\omega_{j}=\sum_{i \in I}\left\langle S_{F}^{-1 / 2} f_{i}, e_{j}\right\rangle Q h_{i}, \quad j \in I .
$$

In this case $F$ obtained as

$$
f_{i}=\sum_{j \in I}\left\langle\omega_{j},\left(Q^{*}\right)^{-1} h_{i}\right\rangle S_{F}^{1 / 2} e_{j}, \quad \forall i \in I .
$$

Relation (2) does not imply, in general, that $F$ is an R-dual of type $I I I$ of $\Omega$, that is, this definition of R-dual is not symmetric. With appropriate choice of $Q$ the symmetry property of the sequences $F$ and $\Omega$ characterized in [8, Theorems 4.4]: 
Theorem 2.3. Let $F$ be a frame for $\mathcal{H}$ and let $\Omega$ be a Riesz sequence with the same optimal bounds as $F$. Denote the synthesis operator for $F$ by $T_{F}$ and the frame operators for $F$ and $\Omega$ by $S_{F}$ and $S_{\Omega}$, respectively. If $\operatorname{dim}\left(\operatorname{ker} T_{F}\right)=\operatorname{dim}\left(\operatorname{span}\left\{\omega_{j}\right\}_{j \in I}^{\perp}\right)$, then there exists orthonormal bases $\left\{e_{i}\right\}_{i \in I}$ and $\left\{h_{i}\right\}_{i \in I}$ for $\mathcal{H}$ such that

$$
\omega_{j}=\sum_{i \in I}\left\langle f_{i}, S_{F}^{-1 / 2} e_{j}\right\rangle \widetilde{S_{\Omega}^{1 / 2}} h_{i}, \quad j \in I,
$$

where $\widetilde{S_{\Omega}^{1 / 2}}$ is an extension of $S_{\Omega}^{1 / 2}$ to an operator on $\mathcal{H}$.

The sequence $\Omega$ defined in (3) is called the symmetrical R-dual of type $I I I$ of $F$ with respect to triple $\left(\left\{e_{i}\right\}_{i \in I},\left\{h_{i}\right\}_{i \in I}, \widetilde{S_{\Omega}^{1 / 2}}\right)$. In this case, for all $i \in I$,

$$
f_{i}=\sum_{j \in I}\left\langle\omega_{j}, \widetilde{S_{\Omega}^{-1 / 2}} h_{i}\right\rangle S_{F}^{1 / 2} e_{j},
$$

that is, $F$ is the R-dual of type $I I I$ of $\Omega$ with respect to triple $\left(\left\{h_{i}\right\}_{i \in I},\left\{e_{i}\right\}_{i \in I}, S_{F}^{1 / 2}\right)$.

\section{Characterizing R-Duality in $\mathcal{H}$}

We first consider symmetrical R-duals of type $I I I$. In [8, Theorems 4.3], it is proved that if $F$ is a frame sequence and $\Omega$ is an R-dual of $F$ of type $I I I$, then the following hold:

(i) $F$ is a frame for $\mathcal{H}$ if and only if $\Omega$ is a Riesz sequence; in the affirmative case the bounds for $F$ are also bounds for $\Omega$,

(ii) $F$ is a Riesz sequence if and only if $\Omega$ is a frame for $\mathcal{H}$; in the affirmative case the bounds for $F$ are also bounds for $\Omega$,

(iii) $\Omega$ is a Riesz Basis if and only if $F$ is a Riesz Basis.

The following section presents another viewpoint of the construction of $R$-duals of type III. In order to develop this viewpoint, we introduce some additional terminology. A map $J: \mathcal{H} \rightarrow \mathcal{H}$ is called antiunitary of $\mathcal{H}$ if $J$ is bijective, conjugate-linear, and isometric. It is called an involution if $J^{2}=I_{\mathcal{H}}$.

The following lemma characterizes antiunitary involutions:

Lemma 3.1. Let $\mathcal{H}$ denote a Hilbert space, and $J_{0}: \mathcal{H} \rightarrow \mathcal{H}$ antiunitary involution.

Let $J: \mathcal{H} \rightarrow \mathcal{H}$ denote a bijection. Then the following are equivalent:

(a) For all $u, v \in \mathcal{H},\langle J u, v\rangle=\overline{\langle u, J v\rangle}$ and $J^{2}=I_{\mathcal{H}}$

(b) $J$ is conjugate-linear and satisfies $J^{2}=I_{\mathcal{H}}$.

(c) There exists a unitary map $U$ such that $J=U J_{0}$, and in addition $J_{0} U J_{0}=U^{*}$.

Proof. Assume (a) For conjugate linearity of $J$, we observe for all $\lambda \in \mathbb{C}$ and all $u, v \in \mathcal{H}$ that by $(a)$,

$$
\langle J \lambda u, v\rangle=\overline{\langle\lambda u, J v\rangle}=\bar{\lambda}\langle J u, v\rangle,
$$

which establishes $J \lambda u=\bar{\lambda} J u$. By analogous reasoning, we get $J(u+v)=J u+J v$, and $(b)$ is established.

For $(b) \Rightarrow(c)$, write $U=J J_{0}$. Then $U$ is isometric, bijective, and linear, hence unitary. Furthermore $U J_{0}=J J_{0}^{2}=J$, and $J^{2}=J_{0}^{2}=I_{\mathcal{H}}$ implies

$$
U J_{0} U J_{0}=J J_{0} J_{0} J J_{0} J_{0}=I_{\mathcal{H}},
$$


and thus $U^{*}=J_{0} U J_{0}$.

$(c) \Rightarrow(a)$ : If $J=U J_{0}$ as in $(c)$, then $J^{2}=U J_{0} U J_{0} U U^{*}=I_{\mathcal{H}}$. Since $J$ is bijective, conjugate-linear and isometric, polarization yields

$$
\langle J u, J w\rangle=\overline{\langle u, w\rangle}
$$

for all $u, v$, and with $w=J v$ and $J^{2}=I_{\mathcal{H}}$ we obtain

$$
\langle J u, v\rangle=\overline{\langle u, J v\rangle} \text {, }
$$

which is $(a)$.

We will explain below that any choice of antiunitary involution gives rise to a construction of $R$-dual sequences that have all the desirable properties of the original construction. The original construction in [1] is based on the following concrete choice of $J_{0}: \ell^{2}(I) \rightarrow \ell^{2}(I)$

$$
J_{0}\left(\left(x_{i}\right)_{i \in I}\right)=\left(\bar{x}_{i}\right)_{i \in I} .
$$

Since each Hilbert space is isometrically isomorphic to some $\ell^{2}(I)$, this example also establishes that antiunitary involutions exist on every Hilbert space $\mathcal{H}$.

The next lemma notes how $J_{0}$, as defined above, enters in the definition of $R$-duals:

Lemma 3.2. With the assumptions of the theorem (2.3), for every $i, j \in I$,

$$
\omega_{j}=T_{\widetilde{H}} o J_{0} o T_{\widetilde{F}}^{*}\left(e_{j}\right),
$$

where, $\widetilde{H}:=\left\{\widetilde{S_{\Omega}^{1 / 2}} h_{i}\right\}_{i \in I}, \quad \widetilde{F}:=\left\{S_{F}^{-1 / 2} f_{i}\right\}_{i \in I}$ and

$$
f_{i}=T_{\widetilde{E}} o J_{0} o T_{\widetilde{\Omega}}^{*}\left(h_{i}\right),
$$

where, $\widetilde{E}:=\left\{S_{F}^{1 / 2} e_{j}\right\}_{j \in I}, \widetilde{\Omega}:=\left\{\widetilde{S_{\Omega}^{-1 / 2}} \omega_{j}\right\}_{j \in I}$.

Proof. By a direct and simple calculation we get (5), which is an operator representation of the symmetrical type $I I I$ of R-duals $\Omega$ and $F$, stated in (3), respectively (4).

Following this example, we define the symmetrical $R$-dual associated to an antiunitary involution $J$, a frame $F$ and a Riesz sequence $\Omega$ by replacing $J_{0}$ with $J$, leading to

$$
\omega_{j}=T_{\widetilde{H}} o J o T_{\widetilde{F}}^{*}\left(e_{j}\right) .
$$

Our aim is to prove that, for any choice of $J$, this construction yields a notion of $R$-dual that has all the desired properties of the original construction.

We will use the following simple Lemma.

Lemma 3.3. Let $U: \ell^{2}(I) \rightarrow \ell^{2}(I)$ is a linear operator and $\widetilde{U}=J o U o J$, where $J$ is an antiunitary involution. Then $\widetilde{U}$ is linear with $\|U\|=\|\widetilde{U}\|$ and $\widetilde{U}^{*}=J o U^{*} o J$.

Proof. $\langle\widetilde{U} f, g\rangle=\langle J o U o J f, g\rangle=\overline{\langle U o J f, J g\rangle}=\overline{\left\langle J f, U^{*} o J g\right\rangle}=\left\langle f, J o U^{*} o J g\right\rangle$.

The next result reads as follows:

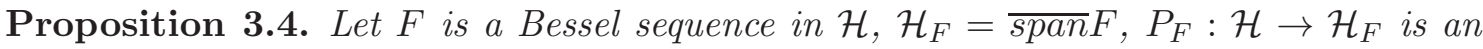
orthogonal projection. Then the following statements hold:

(i) $\widetilde{F}$ is a Riesz sequence if and only if $P_{F} o P^{*} o P_{o} P_{F}: \mathcal{H}_{F} \rightarrow \mathcal{H}_{F}$ is an invertible operator, where $P:=S_{F}^{-1 / 2}$.

(ii) $T_{\Omega}^{*}=J o T_{E}^{*} o T_{\widetilde{F}} o J o T_{\widetilde{H}}^{*}$, and $T_{F}^{*}=J o T_{H}^{*} o T_{\widetilde{\Omega}} o J o T_{\widetilde{E}}^{*}$, where, $E:=\left\{e_{i}\right\}_{i \in I}$ and $H:=\left\{h_{i}\right\}_{i \in I}$. In particular, $\Omega$ is a Bessel sequence in $\mathcal{H}$ if $F$ is a Bessel sequence in $\mathcal{H}$ and vice versa. 
Proof. We first note that $\widetilde{F}:=\left\{P f_{i}\right\}_{i \in I}$ is a Bessel sequence in $\mathcal{H}$ with $T_{\widetilde{F}}^{*}=T_{F}^{*} o P^{*}$, because for $f \in \mathcal{H}$,

$$
\sum_{i \in I}\left|\left\langle f, P f_{i}\right\rangle\right|^{2} \leq B\left\|P^{*} f\right\|^{2}=B\|P\|^{2}\|f\|^{2},
$$

and $T_{\widetilde{F}}^{*} f=\left\{\left\langle f, P f_{i}\right\rangle\right\}_{i \in I}$. In particular, $\widetilde{F}$ is a Bessel sequence, if $T_{\widetilde{F}}^{*}$ is a bounded operator on $\mathcal{H}$. On the other hand for the proof of (i), we have

$$
T_{\widetilde{F}}^{*} o T_{\widetilde{F}}=T_{F}^{*} o P^{*} o P o T_{F}=T_{F}^{*} o P_{F} o P^{*} o P_{o} P_{F} o T_{F}
$$

and $T_{F}: \ell^{2}(I) \rightarrow H_{F}$ is invertible. Hence, $T_{F}^{*}: H_{F} \rightarrow \ell^{2}(I)$ is invertible; then $\widetilde{F}$ is a Riesz sequence if and only if $T_{\widetilde{F}}^{*} o T_{\widetilde{F}}$ is an invertible operator on $\ell^{2}(I)$, if and only if $P_{F} o P^{*} o P_{o} P_{F}$ is an invertible operator on $\mathcal{H}_{F}$, as desired.

(ii) We have

$$
\begin{aligned}
T_{\Omega}^{*} f & =\left\{\left\langle f, \omega_{j}\right\rangle\right\}_{j \in I}=\left\{\left\langle f, T_{\widetilde{H}} o J o T_{\widetilde{F}}^{*}\left(e_{j}\right)\right\rangle\right\}_{j \in I}=\left\{\left\langle T_{\widetilde{H}}^{*} f, J o T_{\widetilde{F}}^{*}\left(e_{j}\right)\right\rangle\right\}_{j \in I} \\
& =\left\{\overline{\left\langle J_{o T_{\overparen{H}}}^{*} f, T_{\widetilde{F}}^{*}\left(e_{j}\right)\right\rangle}\right\}_{j \in I}=\left\{\overline{\left\langle T_{\widetilde{F}} O J_{o} T_{\widetilde{H}}^{*} f, e_{j}\right\rangle}\right\}_{j \in I}=\overline{T_{E}^{*} o\left(T_{\widetilde{F}} o J_{o} T_{\widetilde{H}}^{*} f\right)} \\
& =\left(J o T_{E}^{*} o T_{\widetilde{F}} O J o T_{\widetilde{H}}^{*}\right) f .
\end{aligned}
$$

That is, $T_{\Omega}^{*}=J o T_{E}^{*} o T_{\widetilde{F}} o J o T_{\widetilde{H}}^{*}$. The process is similar for $T_{F}^{*}$.

The next theorem shows that any antiunitary involution can be employed to define a notion of $R$-dual of type III.

Theorem 3.5. Let $F$ is a frame sequence and $\Omega$ is an $R$-dual of type III of $F$ associated to the antiunitary involution $J$. Then the following hold:

$$
\begin{aligned}
T_{\Omega} o T_{\Omega}^{*} & =\left(T_{\widetilde{H}} o J o T_{\widetilde{F}}^{*} o T_{E} o J\right) o\left(J o T_{E}^{*} o T_{\widetilde{F}} o J o T_{\widetilde{H}^{*}}\right) \\
& =T_{\widetilde{H}^{O}} \delta J o T_{\widetilde{F}}^{*} o T_{\widetilde{F}} o J o T_{\widetilde{H}}^{*}
\end{aligned}
$$

and

$$
T_{\Omega}^{*} o T_{\Omega}=\left(J o T_{E}^{*} o T_{\widetilde{F}} o J o T_{\widetilde{H}}^{*}\right) o\left(T_{\widetilde{H}} o J o T_{\widetilde{F}}^{*} o T_{E} o J\right)
$$

Furthermore,

(i) $\Omega$ is a Riesz sequence in $\mathcal{H}$ if and only if $F$ is a frame for $\mathcal{H}$.

(ii) $F$ is a Riesz sequence in $\mathcal{H}$ if and only if $\Omega$ is a frame for $\mathcal{H}$.

Proof. Straightforward computation, using that $J^{2}=I_{\mathcal{H}}, T_{E} o T_{E}^{*}=I_{\mathcal{H}}$, as well as Lemma (3.3) and Proposition (3.4), we have the formulas (8) and (10). In particular,

(i) $\Omega$ is a Riesz sequence if and only if $T_{\Omega}^{*} o T_{\Omega}$ is a bounded and invertible operator on $\ell^{2}(I)$ if and only if

$$
T_{F} o \underbrace{J o T_{\widetilde{H}}^{*} o T_{\widetilde{H}} o J}_{:=U} o T_{F}^{*},
$$

is invertible, (which is by the fact that $S_{F}^{-1 / 2}$ is bounded and invertible). Let now $U:=J o T_{\widetilde{H}}^{*} o T_{\widetilde{H}} o J$. Then $U: \ell^{2}(I) \rightarrow \ell^{2}(I)$ is a positive and invertible operator. Finally, for the proof of proposition, it is enough to show that, $F$ is a frame for $\mathcal{H}$ if and only if $T_{F} o U o T_{F}^{*}: \mathcal{H} \rightarrow \mathcal{H}$ is invertible.

Let $V:=U^{1 / 2}$, then $V$ is a positive and invertible operator on $\ell^{2}(I)$ and we have

$$
T_{F} o U o T_{F}^{*}=\left(V o T_{F}^{*}\right)^{*} o\left(V o T_{F}^{*}\right)
$$


In particular, $F$ is a frame for $\mathcal{H}$ if and only if $T_{F} o T_{F}^{*}: \mathcal{H} \rightarrow \mathcal{H}$ is a bounded and invertible operator, if and only if $T_{F}^{*}: \mathcal{H} \rightarrow \ell^{2}(I)$ is an embedding, if and only if $V o T_{F}^{*}: \mathcal{H} \rightarrow \ell^{2}(I)$ is an embedding, if and only if $\left(V o T_{F}^{*}\right)^{*} o\left(V o T_{F}^{*}\right)=T_{F} o U o T_{F}^{*}$ is an invertible operator, as desired.

(ii) $F$ is a Riesz sequence in $\mathcal{H}$ if and only if $T_{F}^{*} o T_{F}$ is a bounded and invertible operator on $\ell^{2}(I)$ if and only if

$$
T_{\Omega} O \underbrace{J o T_{\widetilde{E}}^{*} o T_{\widetilde{E}} O J}_{:=W} o T_{\Omega}^{*}
$$

is invertible, (which is by the fact that $\widetilde{S_{\Omega}^{1 / 2}}$ is bounded and invertible). Let $W:=J o T_{\widetilde{E}}^{*} o T_{\widetilde{E}} O J$, with a similar proof to $(i), \Omega$ is a frame for $\mathcal{H}$.

The generalization using antiunitary involutions highlights the algebraic and geometric properties of the map $J_{0}$ that lead to the desirable properties of the $R$-dual. Further benefits of this additional freedom of choice in the design of $R$-duals remain to be explored. For example, it is conceivable that a clever choice of $J$ may allow for $R$-duals that fulfill additional symmetry properties.

\section{Representation of $S_{\Omega}$}

In [7], Chuang and Zhao characterized $R$-duals $\Omega$ of of type $I$ of a given frame $F$ by conditions, that are formulated without explicit reference to the construction procedure of such duals. In principle, this result shows that the frame poerator $S_{\Omega}$ is computable from $S_{F}$, although the proof of the theorem is not explicit. The remainder of this section is devoted to a more explicit description of $S_{\Omega}$ in terms of $S_{F}$.

Theorem 4.1. [7] Let $F$ be a frame for $\mathcal{H}$ and $\Omega$ is a Riesz sequence in $\mathcal{H}$. Denote the synthesis operator for $F$ by $T_{F}$, the frame operator of $F$ by $S_{F}$, and the frame operator of $\Omega$ by $S_{\Omega}$. Then $\Omega$ is an $R$-dual of type $I$ of $F$ if and only if the following two conditions hold:

(i) there exists an antiunitary operator $\Lambda: \mathcal{H} \rightarrow \overline{\operatorname{span} \Omega}$ so that $S_{\Omega}=\Lambda S_{F} \Lambda^{-1}$,

(ii) $\operatorname{dim}\left(\operatorname{ker} T_{F}\right)=\operatorname{dim}\left(\left(\operatorname{span}\left\{\omega_{j}\right\}_{j \in I}\right)^{\perp}\right)$.

In the following theorems, we present a representation for the operator $S_{\Omega}$ associated with type $I$ and $I I I$ of $R$-duals. Let now the countable index set $I$ be the integer numbers set $\mathbb{Z}$. We use some ideas of [2].

Theorem 4.2. Let $F$ be a frame for $\mathcal{H}$ and $\Omega$ is a $R$-dual of type $I$ of $F$ with respect to orthonormal bases $\left\{e_{i}\right\}_{i \in I}$ and $\left\{h_{i}\right\}_{i \in I}$. Denote the frame operator of $F$ and $\Omega$ by $S_{F}$ and $S_{\Omega}$, respectively, so that

$$
\sum_{j \in I}\left|\left\langle S_{\Omega} h_{0}, h_{j}\right\rangle\right|<\infty
$$

Then there exist operators $\left\{\mathcal{V}_{j}\right\}_{j \in I}$ on $\mathcal{H}$ so that

(i) $\mathcal{V}_{j}\left(S_{\Omega} h_{0}\right)=S_{\Omega} h_{j}$. 
(ii) If for all $k \in I,\left\{\mathcal{V}_{j}\left(h_{k}\right)\right\}_{j \in I}$ is a Bessel sequence with Bessel bound B, then there exist bounded operators $\left\{\Lambda_{k}\right\}_{k \in \mathbb{Z}}$, which satisfy $\sup _{k \in \mathbb{Z}}\left\|\Lambda_{k}\right\|<\infty$, so that $S_{\Omega}$ associated with $F$ has the representation

$$
S_{\Omega} f=\sum_{i \in \mathbb{Z}}\left\langle f_{i}, f_{0}\right\rangle \Lambda_{i}(f),
$$

with unconditional convergence in the operator norm.

Proof. Let $\mathcal{U}: \mathcal{H} \rightarrow \mathcal{H}$ be unitary shift operator $\mathcal{U} h_{i}:=h_{i+1}$, and let $\mathcal{V}: \mathcal{H} \rightarrow \mathcal{H}$ be the operator defined by $\mathcal{V}=S_{\Omega} \mathcal{U} S_{\Omega}^{-1}$. Then we defined $\left\{\mathcal{V}_{j}\right\}_{j \in I}$ on $\mathcal{H}$ by

$$
\mathcal{V}_{j}:=\mathcal{V}^{j}=S_{\Omega} \mathcal{U}^{j} S_{\Omega}^{-1}
$$

Then (i) is obviously fulfilled, since

$$
\mathcal{V}_{j}\left(S_{\Omega} h_{0}\right)=\mathcal{V}^{j}\left(S_{\Omega} h_{0}\right)=S_{\Omega} \mathcal{U}^{j} h_{0}=S_{\Omega} h_{j}, \quad \forall j \in I .
$$

To proof of (ii), all calculations are similar to proof of theorem 4.5 in [2].

For type $I I I$, the above procedure to computing $\left\langle S_{\Omega} h_{0}, h_{i}\right\rangle$, does not work, and we have change our strategy. First, recall (e.g., [9]) that a representation of $S_{\Omega}$ in terms of simple operators (projections) is the so-called a spectral representation of the operator $S_{\Omega}$.

Let $T: \mathcal{H} \rightarrow \mathcal{H}$ be a bounded self-adjoint linear operator. For $\lambda \in \mathbb{R}$, let $T_{\lambda}=T-\lambda I_{\mathcal{H}}$. Denote the positive square root of $T_{\lambda}^{2}$ by $\left|T_{\lambda}\right|$, the operator $T_{\lambda}^{+}=\frac{1}{2}\left(\left|T_{\lambda}\right|+T_{\lambda}\right)$ which is called the positive part of $T_{\lambda}$, and the operator $T_{\lambda}^{-}=\frac{1}{2}\left(\left|T_{\lambda}\right|-T_{\lambda}\right)$ which is called the negative part of $T_{\lambda}$. In this case, $T_{\lambda}=T_{\lambda}^{+}-T_{\lambda}^{-}$, and the spectral family $\mathcal{E}$ of $T$ is defined by $\mathcal{E}=\left\{E_{\lambda}\right\}_{\lambda \in \mathbb{R}}$, where $E_{\lambda}$ is the projection of $\mathcal{H}$ onto the null space $\mathcal{N}\left(T_{\lambda}^{+}\right)$of $T_{\lambda}^{+}$. Let

$$
m=\inf _{\|x\|=1}\langle T x, x\rangle \text { and } M=\sup _{\|x\|=1}\langle T x, x\rangle
$$

We now have the following theorem, [9, Theorem (9.2-1)]:

Theorem 4.3. Let $T: \mathcal{H} \rightarrow \mathcal{H}$ be a bounded self-adjoint linear operator on a complex Hilbert space $\mathcal{H}$. Then $T$ has the spectral representation as follows:

$$
T=m E_{m}+\int_{m}^{M} \lambda d E_{\lambda},
$$

where the integral is to be understood in the sense of uniform operator convergence. Also, for all $x, y \in \mathcal{H}$,

$$
\langle T x, y\rangle=m W(m)+\int_{m}^{M} \lambda d W(\lambda)
$$

where, $W(\lambda)=\left\langle E_{\lambda} x, y\right\rangle$, and the integral is an ordinary Riemann-Stieltjes integral.

Now we are ready to present a representation for the operator $S_{\Omega}$ associated with type III of $R$-duals.

Theorem 4.4. Let $F$ be a frame for $\mathcal{H}$ with frame operator $S_{F}$, let $\Omega$ be a symmetrical $R$-dual of type III of $F$, with respect to the triple $\left(\left\{e_{i}\right\}_{i \in I},\left\{h_{i}\right\}_{i \in I}, S_{\Omega}^{1 / 2}\right)$, so that

$$
\sum_{j \in I}\left|\left\langle S_{\Omega} h_{0}, h_{j}\right\rangle\right|<\infty .
$$

Assume there exist operators $\left\{\mathcal{V}_{j}\right\}_{j \in I}$ on $\mathcal{H}$ such that

(i) $\mathcal{V}_{j}\left(S_{\Omega} h_{0}\right)=S_{\Omega} h_{j}$, for all $j \in I$. 
(ii) There is a constant $B>0$ so that for all $k \in I$ the set $\left\{\mathcal{V}_{j}\left(h_{k}\right)\right\}_{j \in I}$ is a Bessel sequence, with Bessel bound B.

Then there exist bounded operators $\left\{\Lambda_{i}\right\}_{i \in \mathbb{Z}}$, which satisfy $\sup _{i \in \mathbb{Z}}\left\|\Lambda_{i}\right\|<\infty$, and constants $\mathcal{C}_{i}$, so that $S_{\Omega}$ associated with $F$ has the representation

$$
S_{\Omega} f=\sum_{i \in I}\left(M\left\langle S_{F}^{-1 / 2} f_{i}, S_{F}^{-1 / 2} f_{0}\right\rangle-\mathcal{C}_{i}\right) \Lambda_{i}(f)
$$

with unconditional convergence in the operator norm.

Proof. Since $S_{\Omega}$ is a bounded self-adjoint and linear operator on $\mathcal{H}$, by the equation (14) we have

$$
\begin{aligned}
\left\langle S_{\Omega} h_{0}, h_{i}\right\rangle & =m W(m)+\int_{m}^{M} \lambda d W(\lambda) \\
(\text { Riemann-Stieltjes integral }) & =m W(m)+M W(M)-m W(m)-\int_{m}^{M} W(\lambda) d \lambda,
\end{aligned}
$$

where $W(\lambda)=\left\langle E_{\lambda} h_{0}, h_{i}\right\rangle$ and $E_{\lambda}$ is the projection of $\mathcal{H}$ onto the null space $\mathcal{N}\left(S_{\Omega, \lambda}^{+}\right)$of $S_{\Omega, \lambda}^{+}$, with $S_{\Omega, \lambda}=S_{\Omega}-\lambda I$. Therefore,

$$
\left\langle S_{\Omega} h_{0}, h_{i}\right\rangle=M W(M)-\int_{m}^{M} W(\lambda) d \lambda
$$

On the other hand, $\Omega$ is a Riesz sequence in $\mathcal{H}$ and then it is a Riesz basis for $V:=\overline{\operatorname{span}} \Omega$. Then the sequence $\left\{S_{\Omega}^{-1 / 2} \omega_{j}\right\}_{j \in I}$ is an orthonormal basis for $V$ [8, Lemma (1.1)]; (note that $\left.\mathcal{N}\left(S_{\Omega, \lambda}^{+}\right) \subset V\right)$. Consider the extension $\widetilde{S_{\Omega}^{-1 / 2}}$ of $S_{\Omega}^{-1 / 2}$ to an operator on $\mathcal{H}$, as in [8, Lemma (1.3)]; $\left\{\widetilde{S_{\Omega}^{-1 / 2}} \omega_{j}\right\}_{j \in I}$ is an orthonormal basis for $V$, too. Therefore, the orthogonal projection $E_{M}$ of $\mathcal{H}$ onto $\mathcal{N}\left(S_{\Omega, \lambda}^{+}\right)$is given by

$$
E_{M} f=\sum_{j \in I}\left\langle f \widetilde{S_{\Omega}^{-1 / 2}} \omega_{j}\right\rangle \widetilde{S_{\Omega}^{-1 / 2}} \omega_{j}, \quad \forall f \in \mathcal{H}
$$

It is enough to prove that if we define $E_{M}$ by (18), then $E_{M} f=f$ for $f \in \mathcal{N}\left(S_{\Omega, \lambda}^{+}\right)$ and $E_{M} f=0$ for $f \in\left(\mathcal{N}\left(S_{\Omega, \lambda}^{+}\right)\right)^{\perp}$; the first equation follows by the orthonormality of $\left\{S_{\Omega}^{-1 / 2} \omega_{j}\right\}_{j \in I}$, and the second by the fact that the range of $S_{\Omega}^{-1 / 2}$ equals $\mathcal{N}\left(S_{\Omega, \lambda}^{+}\right)$because $S_{\Omega}^{-1 / 2}$ is bijection on $\mathcal{N}\left(S_{\Omega, \lambda}^{+}\right)$. Therefore

$$
\left.\left\langle E_{M} h_{0}, h_{i}\right\rangle=\sum_{j \in I}\left\langle h_{0}, \widetilde{S_{\Omega}^{-1 / 2}} \omega_{j}\right\rangle \widehat{\left\langle S_{\Omega}^{-1 / 2}\right.} \omega_{j}, h_{i}\right\rangle
$$

(by equation (4)) $=\left\langle S_{F}^{-1 / 2} f_{i}, S_{F}^{-1 / 2} f_{0}\right\rangle$. 
We now have

$$
\begin{aligned}
S_{\Omega} f & =\sum_{j \in I}\left\langle f, h_{j}\right\rangle S_{\Omega}\left(h_{j}\right) \\
& =\sum_{j \in I}\left\langle f, h_{j}\right\rangle \mathcal{V}_{j}\left(S_{\Omega}\left(h_{0}\right)\right) \\
& =\sum_{j \in I}\left\langle f, h_{j}\right\rangle \mathcal{V}_{j}\left(\sum_{i \in I}\left\langle S_{\Omega}\left(h_{0}\right), h_{i}\right\rangle h_{i}\right) \\
& =\sum_{i \in I}\left\langle S_{\Omega} h_{0}, h_{i}\right\rangle \sum_{j \in I}\left\langle f, h_{j}\right\rangle \mathcal{V}_{j}\left(h_{i}\right) \\
& =\sum_{i \in I}\left(M\left\langle E_{M} h_{0}, h_{i}\right\rangle-\mathcal{C}_{i}\right) \sum_{j \in I}\left\langle f, h_{j}\right\rangle \mathcal{V}_{j}\left(h_{i}\right) \\
& =\sum_{i \in I}\left(M\left\langle S_{F}^{-1 / 2} f_{i}, S_{F}^{-1 / 2} f_{0}\right\rangle-\mathcal{C}_{i}\right) \sum_{j \in I}\left\langle f, h_{j}\right\rangle \mathcal{V}_{j}\left(h_{i}\right)
\end{aligned}
$$

where $\mathcal{C}_{i}:=\int_{m}^{M} W(\lambda) d \lambda$. Defining $\Lambda_{i}$ by $\Lambda_{i}(f)=\sum_{j \in I}\left\langle f, h_{j}\right\rangle \mathcal{V}_{j}\left(h_{i}\right)$, then we have

$$
S_{\Omega} f=\sum_{i \in I}\left(M\left\langle S_{F}^{-1 / 2} f_{i}, S_{F}^{-1 / 2} f_{0}\right\rangle-\mathcal{C}_{i}\right) \Lambda_{i}(f)
$$

as desired. For convergence, the procedure is almost the same as Theorem 4.5 in [2], but we are reviewing it. The sequence $\left\{\mathcal{V}_{j}\left(h_{i}\right)\right\}_{j \in I}$ is a Bessel sequence with bound $B$, then for any finite set $J \subset I$,

$$
\left\|\sum_{j \in J}\left\langle f, h_{j}\right\rangle \mathcal{V}_{j}\left(h_{i}\right)\right\|^{2} \leq B \sum_{j \in J}\left|\left\langle f, h_{j}\right\rangle\right|^{2}=B\|f\|^{2}
$$

that is, the series defining $\Lambda_{i}$ are unconditionally convergent and $\left\|\Lambda_{i}\right\| \leq \sqrt{B}, \forall k \in \mathbb{Z}$. On the other hand, for finite subsets $I_{1}$ and $I_{2}$ of $I$, from the above calculations,

$$
\begin{aligned}
& \left\|\sum_{i \in I_{1}}\left(M\left\langle S_{F}^{-1 / 2} f_{i}, S_{F}^{-1 / 2} f_{0}\right\rangle-\mathcal{C}_{i}\right) \sum_{j \in I_{2}}\left\langle f, h_{j}\right\rangle \mathcal{V}_{j}\left(h_{i}\right)\right\| \\
& =\left\|\sum_{i \in I_{1}}\left\langle S_{\Omega} h_{0}, h_{i}\right\rangle \sum_{j \in I_{2}}\left\langle f, h_{j}\right\rangle \mathcal{V}_{j}\left(h_{i}\right)\right\| \\
& \leq\left\{\sum_{i \in I_{1}}\left|\left\langle S_{\Omega} h_{0}, h_{i}\right\rangle\right|\right\}\left\|\sum_{j \in I_{2}}\left\langle f, h_{j}\right\rangle \mathcal{V}_{j}\left(h_{i}\right)\right\| \\
& \leq\left\{\sum_{i \in I_{1}}\left|\left\langle S_{\Omega} h_{0}, h_{i}\right\rangle\right|\right\} \sqrt{B}\|f\| .
\end{aligned}
$$


By the convergence of the series (11), it follows that the series in the construction of $\Lambda_{i}$ are unconditionally convergent. Finally, for a finite subset $J \subset I$, we have

$$
\begin{aligned}
\| S_{\Omega} & -\sum_{i \in J}\left(M\left\langle S_{F}^{-1 / 2} f_{i}, S_{F}^{-1 / 2} f_{0}\right\rangle-\mathcal{C}_{i}\right) \Lambda_{i} \| \\
& =\sup _{\|f\|=1}\left\|S_{\Omega}(f)-\sum_{i \in J}\left(M\left\langle S_{F}^{-1 / 2} f_{i}, S_{F}^{-1 / 2} f_{0}\right\rangle-\mathcal{C}_{i}\right) \Lambda_{i}(f)\right\| \\
& =\sup _{\|f\|=1}\left\|\sum_{i \in J^{c}}\left(M\left\langle S_{F}^{-1 / 2} f_{i}, S_{F}^{-1 / 2} f_{0}\right\rangle-\mathcal{C}_{i}\right) \Lambda_{i}(f)\right\| \\
& \leq\left\{\sum_{i \in J^{c}}\left|\left(M\left\langle S_{F}^{-1 / 2} f_{i}, S_{F}^{-1 / 2} f_{0}\right\rangle-\mathcal{C}_{i}\right)\right|\right\} \sup _{\|f\|=1} \sup _{i \in J^{c}}\left\|\Lambda_{i}(f)\right\| \\
& \leq\left\{\sum_{i \in J^{c}} \mid\left(M\left\langle S_{F}^{-1 / 2} f_{i}, S_{F}^{-1 / 2} f_{0}\right\rangle-\mathcal{C}_{i} \mid\right)\right\} \sqrt{B} .
\end{aligned}
$$

Now using (11) and that $\left|\left\langle S_{\Omega} h_{0}, h_{i}\right\rangle\right|=\left|\left(M\left\langle S_{F}^{-1 / 2} f_{i}, S_{F}^{-1 / 2} f_{0}\right\rangle-\mathcal{C}_{i}\right)\right|$, it follows that the operators converge to $S_{\Omega}$ unconditionally in the operator norm.

\section{REFERENCES}

[1] Casazza, P. G., Kutyniok, G. and Lammers M.: Duality principles in frame theory. J. Fourier Anal. Appl. 10, 383-408 (2004)

[2] Casazza, P. G., Kutyniok, G. and Lammers M.: Duality principles, localization of frames, and Gabor theory, In: Papadakis, M., Laine, A.F., Unser, M.A.(eds).SPIE Proceedings in Wavelets XI, Vol. 5914 (2005).

[3] Casazza, P. G.: Modern tools for Weyl-Heisenberg ( Gabor ) frame theory, Adv. in Imag. and Electron. Physics, 115, 1-127 (2001)

[4] Christensen, O.: Frame and Bases, An Introductory Course, Birkhäuser, Basel (2008)

[5] Christensen, O., Kim H. O. and Kim R. Y.: On the duality principle by Casazza, Kutyniok, and lammers, J. Fourier Anal. Appl. 17, 640-655 (2011)

[6] Christensen, O., Xiao, XC. and Zhu, YC.: Characterizing R-duality in Banach spaces, Acta Math. Sin. Engl. Ser. 1, 75-84 (2013)

[7] Chuang, Z., Zhao, J.: On equivalent conditions of two sequences to be R-dual, J. Inequal. Appl. 10, $1-8(2015)$

[8] Stoeva, D. T. and Christensen, O.: On R-duals and the Duality principle in Gabor Analysis, J. Fourier Anal. Appl. 21, 383-400 (2015)

[9] Kreyszig, E.: Introductory Functionl Analysis with approximation, John-Wiley, New York, (1978) 